\title{
Clustered Regression Analysis
}

\author{
David Lindgren, Lennart Ljung \\ Division of Automatic Control \\ Department of Electrical Engineering \\ Linköpings universitet, SE-581 83 Linköping, Sweden \\ WWW: http://www. control.isy.liu.se \\ E-mail: david@isy.liu.se, ljung@isy.liu.se
}

16th August 2002

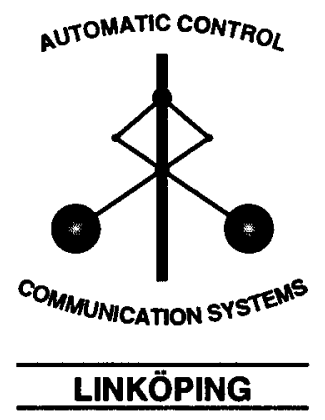

Report no.: LiTH-ISY-R-2456

Submitted to CDC02

Technical reports from the Control \& Communication group in Linköping are available at http://www. control.isy.liu.se/publications. 


\begin{abstract}
Cluster structure in (multicollinear) data can be utilized by pattern recognition methods in order to find adequate subspaces for nonlinear regression. When regressing a particular severely nonlinear function, it is demonstrated that this approach is superior to polynomial PLS. It is also demonstrated that for nonlinear functions, the choice of regressing explained variables onto the explaining variables, or vice-versa, is not arbitrary. Numerical experiments indicate that the classical statistical model is more powerful than the inverse regression approach.
\end{abstract}

Keywords: Nonlinear regression, Subspace regression, Inverse regression 


\title{
Clustered Regression Analysis ${ }^{1}$
}

\author{
David Lindgren ${ }^{2} \quad$ Lennart Ljung \\ Division of Automatic Control \\ Linköpings universitet, Sweden
}

\begin{abstract}
Cluster structure in (multicollinear) data can be utilized by pattern recognition methods in order to find adequate subspaces for nonlinear regression. When regressing a particular severely nonlinear function, it is demonstrated that this approach is superior to polynomial PLS. It is also demonstrated that for nonlinear functions, the choice of regressing explained variables onto the explaining variables, or vice-versa, is not arbitrary. Numerical experiments indicate that the classical statistical model is more powerful than the inverse regression approach.
\end{abstract}

\section{Introduction}

The background to this work is the regression problem associated with concentration measurements with the electronic tongue (ET) [11]. A measurement vector $x_{i} \in \mathbb{R}^{n}$ from the ET is very large, typically in the order $n=10000$. With each measurement $x_{i}$ is associated a scalar concentration $y_{i}$ in terms of moles. We wish to find a function $f$ that with highest possible accuracy relates the vectors $x_{i}$ to the concentrations $y_{i}$, that is, the expected magnitude of the error $e_{i}$ in

$$
y_{i}=f\left(x_{i}\right)+e_{i}
$$

should be minimal. The error $e_{i}$ is generally due to nonperfect $f$ and to a random measurement noise sample $\epsilon_{i}$ which we cannot account for. Of course, for the relation $f$ (and the ET) to be useful, we must be able to state that the expected magnitude of $e_{i}$ is small when a real measurement is made and $y_{i}$ is estimated.

An appropriate statistical model for measurements is

$$
x_{i}=\phi\left(y_{i}, \eta\right)+\epsilon_{i}
$$

where the function $\phi(y, \eta)$ for a fixed parameter $\eta$ describes an $n$-dimensional trajectory as $y$ increases from low to high values (concentrations), $\phi: \mathbb{R} \rightarrow \mathbb{R}^{n}$. The noise sample $\epsilon_{i}$ is taken from a distribution assumed to have zero mean and (unknown) covariance matrix $\Sigma$.

\footnotetext{
${ }^{1}$ This work is supported by the Swedish Research Council.

${ }^{2}$ Corresponding author, david@isy.liu.se.
}

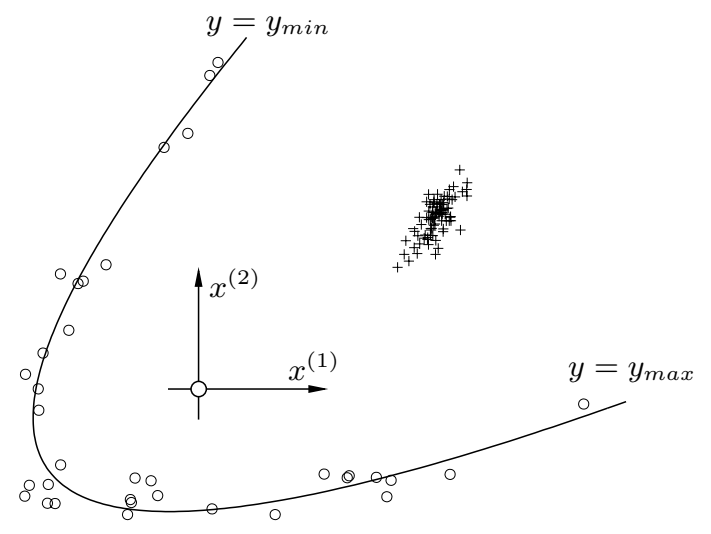

Figure 1: A trajectory $\phi(y)$ together with a number of noise obscured measurements (o) in two dimensions $\left(x^{(1)} / x^{(2)}\right)$. The cluster illustrates the noise distribution $(+)$.

Here we also assume that there are no temporal correlation between the noise samples; $\mathrm{E}\left[\epsilon_{i} \epsilon_{j}\right]=0$ when $j \neq i$. Figure 1 gives an example of a bivariate, nonlinear trajectory and a number of possible measurements $x_{i}$. Note that $x^{(j)}$ is the $j$ th entry of the vectors $x_{i}$.

The ordinary steps to solve the regression problem above are first to select a suitable parameterization of $\phi(y)$, and second to estimate the parameter $\eta$ from an estimation (training) data set $\left\{x_{i}, y_{i}\right\}_{1}^{N}$. The parameterization could for instance be polynomial, and $\eta$ the parameter matrix where the entries are the coefficients of the polynomials. Given a parameterization, the maximum likelihood (ML) estimate of $\eta$ in (2) is expressed as

$$
\begin{aligned}
\eta_{\mathrm{ML}} & =\arg \min _{\eta}\left|\Sigma_{\mathrm{ML}}(\eta)\right| \\
& =\arg \min _{\eta}\left|\sum_{i=1}^{N}\left(\phi\left(y_{i}, \eta\right)-x_{i}\right)\left(\phi\left(y_{i}, \eta\right)-x_{i}\right)^{T}\right|
\end{aligned}
$$

assuming normally distributed noise [7]. The least squares (LS) estimate is expressed similarly; replace the matrix determinant $\left|\Sigma_{\mathrm{ML}}(\eta)\right|$ above with the trace operator $\operatorname{tr}\left[\Sigma_{\mathrm{ML}}(\eta)\right]$.

A complication arise when the (large) $n$-by- $n$ covariance matrix $\Sigma_{\mathrm{ML}}(\eta)$ is rank deficient, which is always the case when $N<n$. Then $\left|\Sigma_{\mathrm{ML}}(\eta)\right|=0$ for every 
possible choice of parameter, and (3) cannot be used at all to find $\eta$. To circumvent this complication, one often has to utilize the fact that the nonlinear trajectory mainly resides within a linear $k$-dimensional subspace, where $k \ll n($ and $k<N)[9,2,21]$. Thus, we can factorize $\phi$ as $\phi(y, \eta)=S \phi_{0}\left(y, \eta_{0}\right)$ for some orthonormal $n$-by- $k$ matrix $S$. Hence the model (2) evolves as

$$
x_{i}=S \phi_{0}\left(y_{i}, \eta_{0}\right)+\epsilon_{i} .
$$

The parameter estimation is then conducted in the subspace spanned by the columns of $S$, that is, one solves

$$
\min _{\eta_{0}}\left|\sum_{i=1}^{N}\left(\phi_{0}\left(y_{i}, \eta_{0}\right)-S^{T} x_{i}\right)\left(\phi_{0}\left(y_{i}, \eta_{0}\right)-S^{T} x_{i}\right)^{T}\right| .
$$

Thus, if we know $S$ it is possible to estimate $\eta_{0}$ and the subspace noise covariance matrix $S^{T} \Sigma S$, even when $N<n$. Alternatively, the regression (1) can be solved directly in LS-sense by letting $f(x)=g\left(S^{T} x, \theta\right)$ for some appropriate function family $g$.

However, the subspace $S$ is seldom known; it has to be found by calculations on empirical data. For the estimation of the parameters of a nonlinear $\phi$ it is of course particularly important to find the minimal dimensionality $(k)$ that well describes $\phi$ (or $f$ ). This is difficult for multicollinear data sets (linearly dependent $x^{(j)}$ 's) for which $n>N$ is typical [26].

\subsection{Subspace Techniques}

There are many well-known and robust techniques to find a subspace $S$ in which the actual linear or nonlinear regression can take place. One of the simplest is to use a limited number of uncorrelated principal components chosen after an error-impact or a variance criterion (PCR) [17]. QR-decomposition is a similar way to orthogonalize the problem [8]. One popular technique in chemometrics is the Partial Least Squares algorithm (PLS), which also can be formulated as a subspace regression method. In the original PLS algorithm, a set of linear combinations of the variables $x^{(j)}$ that have maximum covariance with the variable $y$ is calculated iteratively and used in the regression [25]. It has been shown, though, that the PLS-subspace can be found by a QR-decomposition of a Krylov matrix composed from the matrix $\left[x_{i}\right]_{1}^{p}$ and the vector $\left[y_{i}\right]_{1}^{p}[20]$. A nonlinear extension to PLS is the polynomial PLS, which uses a polynomial inner relation between $x$ and $y$ [24]. Instead of maximizing the covariance between $x$ and $y$ as in PLS, the criterion could be correlation, as in Canonical Correlation Analysis (CCA) [13] (does not work well applied directly to multicollinear data). In $[22,2]$ it is described how the ML subspace is calculated (not for multicollinear data). Projection pursuit regression (PPR) [5] iteratively finds the directions of the subspace, one at a time, that reduce the (residual) unexplained variance as much as possible.

\subsection{Clustered Data Sets}

The observations or measurements of a data set can be partitioned into a number of categories or clusters. Clustered measurement data are not unnatural. Assume we want to calibrate an instrument on concentrations. Then it is natural to subject the instrument to a set of known concentrations - say we measure 20 times on each of 10 concentrations within an interesting range. This results in a clustered data set.

A number of ways to utilize the clustering of data to improve the regression have been proposed. Sliced Inverse Regression (SIR) [16] divides the range of $y$ into a number of intervals. Each interval identifies a cluster. A PCA on the cluster means is then used as a subspace for regression. Fitting local linear models to data partitions are proposed in [1] and clustering in conjunction with Support Vector Machines (SVM) has been exploited in [4].

In this paper the regression subspace will be optimized for accurate classification, as will be described below. This approach is the main contribution of the paper and it has not been seen elsewhere. We denote the method Clustered Regression Analysis (CRA).

\section{Inverse vs. Classical Regression}

When creating the regression function $f$ in (1), there are basically two approaches at hand:

1. Let $f(x)=g(x, \theta)$ for an adequate function family $g$, and let $\theta$ minimize some loss function of the errors $e_{i}$ for an estimation data set $\left\{x_{i}, y_{i}\right\}_{1}^{p}$. This approach is denoted inverse regression.

2. Chose a function family $\phi(y, \eta)$, solve (3) (for normally distributed noise) and derive the conditional probability $p(y \mid x)$. Estimate $y$ as $f(x)=\mathrm{E}[y \mid x]$ or as $f(x)=\arg \max _{y} p(y \mid x)$. This approach is denoted classical regression.

The first approach is straightforward and gives a function $f$ that explicitly maps a new measurement to an estimation of $y$. In the second, the estimation of $y$ is formulated implicitly by the model, for instance as the conditional expectation $f(x)=\mathrm{E}[y \mid x]$ or as the conditional ML-value $f(x)=\arg \max _{y} p(y \mid x)$. The determination of the conditional probability $p(\cdot \mid \cdot)$ is essential and requires that the noise distribution is known.

There is no final answer which method to use to calibrate an instrument. Even in the linear case the question is still open. The ongoing discussion started back in 1939 by C. Eisenhart [3] who advocated the classical approach. In 1967 R. C. Krutchkoff [14] 

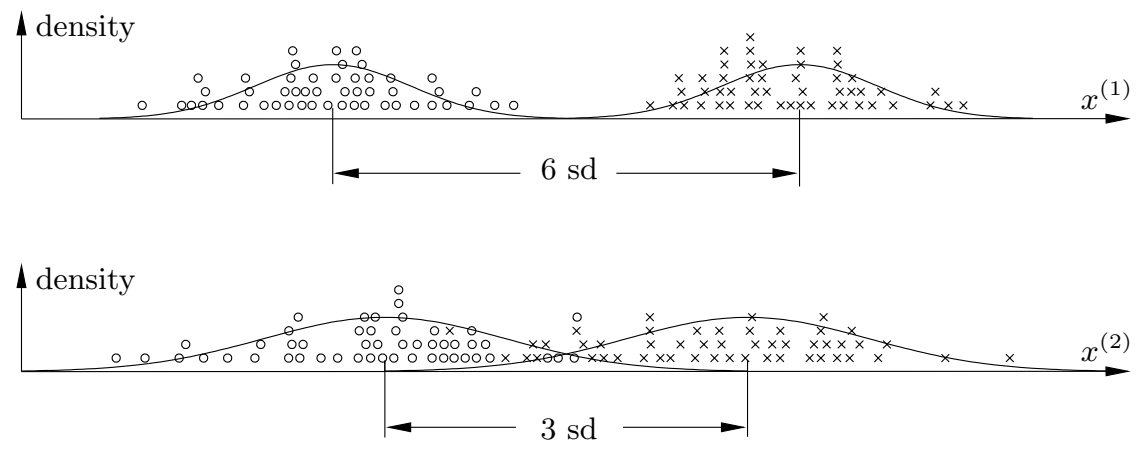

Figure 3: Two dot diagrams of two univariate clusters. For the variable $x^{(1)}$ the cluster distance is 6 standard deviations $(\mathrm{sd})$, and for $x^{(2)}$ only $3 \mathrm{sd}$. If the dots in the diagrams are noisy measurements on two concentrations, it is obvious that $x^{(1)}$ measures the concentration with much higher accuracy than $x^{(2)}$.

Table 1: Validation $\operatorname{RMSE}=\sqrt{\operatorname{mean}\left[(y-\hat{y})^{2}\right]}$ for different methods and noise levels $\sigma^{2}, 1000 \times \operatorname{mean} \pm$ std for 30 data sets. Linear and $3^{\text {rd }}$ order polynomial fit. ML, Bayes, and inverse regression.

\begin{tabular}{|c|c|c|c|c|c|c|}
\hline$\sigma^{2}$ & ML, $3^{\text {rd }}$ ord. pol. & Bayes, $3^{\text {rd }}$ ord. pol. & ML, linear & Bayes, linear & Inverse, $3^{\text {rd }}$ ord. pol. & Inverse, linear \\
\hline \hline 1 & $30 \pm 11$ & $29 \pm 10$ & $44 \pm 22$ & $44 \pm 22$ & $46 \pm 27$ & $44 \pm 22$ \\
\hline 5 & $59 \pm 32$ & $54 \pm 27$ & $88 \pm 46$ & $86 \pm 44$ & $94 \pm 50$ & $87 \pm 45$ \\
\hline 10 & $85 \pm 45$ & $76 \pm 35$ & $125 \pm 55$ & $120 \pm 50$ & $126 \pm 56$ & $124 \pm 53$ \\
\hline 50 & $179 \pm 94$ & $156 \pm 76$ & $204 \pm 91$ & $188 \pm 78$ & $250 \pm 141$ & $197 \pm 84$ \\
\hline
\end{tabular}

conducted extensive Monte Carlo simulations on linear univariates, indicating the superiority of the inverse approach. After that, the theory has evolved in e.g. $[10,12,19,15,23]$, but the treatment is mainly limited to linear problems. The subspaces we shall study below contain potentionally very nonlinear trajectories, why a simulation study is motivated that indicate which regression model to use in our case.

\subsection{Classical Estimation of $y$}

Two possible estimators for classical models are the conditional expectation (Bayes):

$$
\hat{y}_{\text {Bayes }}=\mathrm{E}[y \mid x]=\frac{\int y p(y \mid x) d y}{\int p(y \mid x) d y},
$$

and the ML:

$$
\hat{y}_{\mathrm{ML}}=\arg \max _{y} p(y \mid x)
$$

For normally distributed noise the conditional probability is, given the function $\phi$ and the noise covariance matrix $\Sigma$,

$$
p(y \mid x)=\frac{e^{-(\phi(y)-x)^{T} \Sigma^{-1}(\phi(y)-x) / 2}}{(2 \pi)^{\frac{n}{2}}|\Sigma|^{\frac{1}{2}}},
$$

c.f. the multivariate normal pdf. In practice, (5) and (6) are solved numerically with the prior $y_{\min } \leq y \leq$ $y_{\max }$. Note that the ML and Bayes estimates not necessarily are equal, which in theory is the case for linear estimation in normally distributed noise.

\subsection{Monte Carlo Simulation}

In this section the performance of classical and inverse regression will be compared when the trajectory is very nonlinear. The trajectory is modeled by polynomial $g$ and $\phi ; g(x, \theta)=\theta^{T} \mathcal{P}_{c}(x)$ and $\phi(y, \eta)=\eta 1_{n} \mathcal{P}_{c}(y)$. $\mathcal{P}_{c}$ is the power expansion operator that generates all powers and distinct mixed products up to order c. For instance, $\mathcal{P}_{2}(a)=\left[\begin{array}{ll}a & a^{2}\end{array}\right]$ and $\mathcal{P}_{2}\left(\left[\begin{array}{ll}a & b\end{array}\right]\right)=$ $\left[\begin{array}{lllll}a & a^{2} & a b & b^{2} & b\end{array}\right]$ (similarly for column vectors). $1_{n}$ is an $n$-vector with ones, $1_{n}=\left[\begin{array}{llll}1 & 1 & \ldots & 1\end{array}\right]^{T}$. The parameter vector $\theta$ and matrix $\eta$ are easy to estimate from a training set, since they enter the functions linearly. In this study we shall focus on the regression and postpone the treatment of the subspace problem to the next section. The regression will thus be restricted to two variables.

Artificial data will be generated according to the statistical model (2). The trajectory $\phi_{0}(y)$ will be:

$$
\phi_{0}(y)=\left[\begin{array}{c}
\sin \pi y \\
\sin 1.7 y
\end{array}\right], \quad-1<y<1 .
$$

This particular function is not typical in any way, it is just nonlinear and also non-uniquely invertible in the first component $x^{(1)}$. A number of batches of data sets will be tested, with different noise variance magnitude in each batch (normal distributed noise). In every batch there are 30 data sets, where the noise covariance matrix is taken randomly as $\Sigma=\sigma^{2} \cdot n n^{T}$. $n$ is a 2 -by-2 matrix where each entry is a sample of the normal distribution $N(0,1)$. Thus, the trajectory is fixed, but the 
noise covariance is random for every data set. In every data set there are 21 measurements used for estimation (training) of $\theta / \eta / \Sigma$ and 61 for validation in terms of root mean square error, $\operatorname{RMSE}=\sqrt{\operatorname{mean}\left[(y-\hat{y})^{2}\right]}$. Figure 2 (left) is a sample of a data set generated as described above. The integrals in (5) are numerically approximated by the sum of 200 points in the interval $-1 \leq y \leq 1$. The optimization in (6) is solved by taking the best of 200 tested values of $y$ in the same interval.

Table 1 summarizes the result of the simulation. It appears that inverse regression with polynomial fit should be avoided. In every instance, the classical regression with Bayes prediction is best. The conclusion is that the inverse regression model with polynomial fit is not versatile enough to adapt to a trajectory like (8), while the classical regression model with polynomial fit greatly improves the linear regression. Baysian estimation is to be preferred.

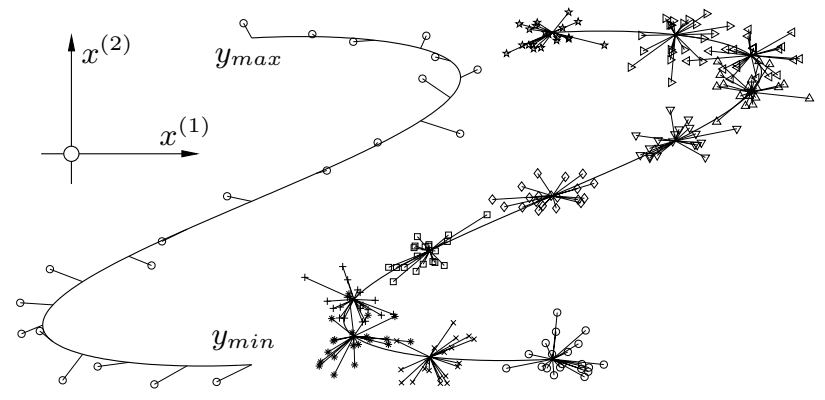

Figure 2: The nonlinear trajectory (8) plotted; to the left with 1 and to the right with 21 measurements in for each distinct $y$. The right hand plot is a projection of $\mathbb{R}^{10}$. The simulated measurements are generated by the model (2). The $y$ 's are uniformly distributed in the interval $y_{\min }$ to $y_{\max }$.

\section{Using Clusters to Find a Subspace}

The idea behind Clustered Regression Analysis (CRA) is to use structure in data to improve the nonlinear regression. The basic assumption is that the data are clustered. The clustering can emanate from measurements on a finite number of objects or concentrations (intervals), or it could be different operating regimes of a dynamical system. Methods from the area of pattern recognition [6] is then used to find the subspace $S$ where the nonlinear regression is conducted.

The prime interest in pattern recognition is to discriminate clusters in order to make accurate classification. Particularly interesting are statistical intercluster distances, which are used to measure the ability to discriminate one cluster from another, for instance the Mahalanobis distance with unit standard deviations
[18]. Large distance means high potential classification accuracy, and this accuracy also reflects the regression accuracy in the region between the clusters, since it is a relative measure of the noise magnitude. This is illustrated by Figure 3. The inter-cluster distances and ultimately the classification accuracy can thus in a very general (nonlinear) way express the regression quality of a subspace $S$.

\subsection{Artificial Example}

In this section, regression in subspaces obtained from a pattern recognition method will be compared to PLS when the variate is 10 -dimensional. The pattern recognition method we shall use calculates the $k$-dimensional linear subspace $S$ where the classification accuracy is approximately and locally optimized. By intuition at least, this objective should be beneficial also for regression problems, as motivated. The pattern recognition method is the ODP (Optimal Discriminative Projection, patented calibration technique) [18], which combines sophisticated model optimization with regularization to handle multicollinearity. The technique to optimize the regression subspace for classification accuracy and then use a nonlinear, classical regression model to estimate $y$, we denote Clustered Regression Analysis (CRA).

Artificial data will be generated about the same way as was described in Section 2.2. However, this time a number of discrete $y$ 's are used in the training set, and for each one, a number of noise obscured $x$ 's are generated. The range of $y$ is divided into 11 clusters and in each cluster there is 20 data points. The trajectory is still restricted to 2 dimensions, but mixed into a 10-dimensional space with noise according to (4). See Figure 2 (right) for a sample of a 2-dimensional projection.

A total of 4 regression methods are compared: ordinary linear regression in 10 dimensions, $5^{\text {th }}$ order polynomial PLS with 2 latent variables (PLS-2/5), $2^{\text {nd }}$ order polynomial PLS with 5 latent variables (PLS-5/2), and classical $5^{\text {th }}$ order polynomial regression in a 2-dimensional ODP subspace with ML-estimation (CRA). The validation data are not subjected to the clustering, but uniformly spread over the range.

3.1.1 Results: The regression errors (RMSE) are given in Table 2. For reasonable noise levels, CRA gives better or much better regression accuracy. In Figure 4 the subspaces obtained from ODP and PLS are compared for an instance with particularly high noise level in the non-trajectory subspace (orthogonal to $S$ ). The plots are due to clustered validation data. Apparently, the noise level in the OPD subspace is significantly lower than in the PLS subspace. 


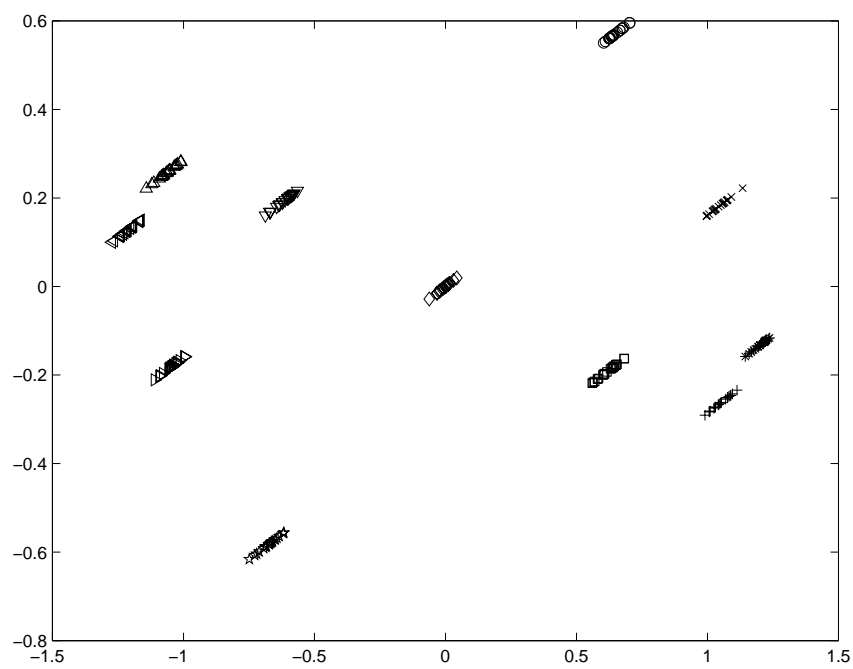

$(\mathrm{ODP})$

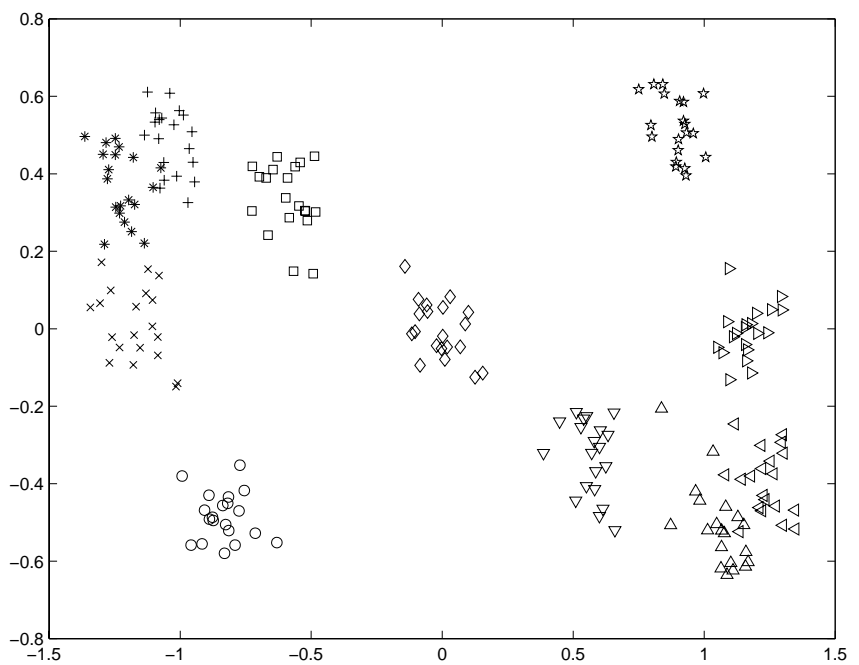

(PLS)

Figure 4: The ODP subspace compared to the PLS, clustered validation data. The regression accuracy for $5^{\text {th }}$ order polynomial classical regression in the ODP subspace is much higher (RMSE $=9.6 \cdot 10^{-3}$ ) compared to the PLS $\left(\mathrm{RMSE}=39 \cdot 10^{-3}\right)$.

Table 2: Validation RMSE for different $\sigma^{2}$, 10-dimensional case, $1000 \times$ mean \pm std for 10 data sets, for CRA (see text), linear regression, and polynomial PLS with latent variables/polynomial order.

\begin{tabular}{|l|l|l|l|l|}
\hline$\sigma^{2}$ & CRA & Linear & PLS-2/5 & PLS-5/2 \\
\hline 2.5 & $30 \pm 17$ & $52 \pm 23$ & $124 \pm 23$ & $69 \pm 25$ \\
10 & $73 \pm 31$ & $121 \pm 33$ & $223 \pm 50$ & $154 \pm 42$ \\
40 & $175 \pm 96$ & $176 \pm 78$ & $279 \pm 66$ & $216 \pm 71$ \\
\hline
\end{tabular}

\section{Electronic Tongue Data}

In this section, the CRA and polynomial PLS methods are tested on real sensor data from an electronic tongue (ET). The ET is subjected to different concentrations of a chemical. Every concentration of $0,3.1$, $3.7,4.5,5.2,5.6,6.9,7.5,8,8.8,9.0,10.6,10.8 \mathrm{mM}$ are measured upon 20 times, $x_{i} \in \mathbb{R}^{403}$.

\subsection{Random Validation}

The data set classes are randomly divided into estimation and validation data at the ratio 75/25. Model parameters are estimated from the estimation data and regression error $\mathrm{RMSE}=\sqrt{\operatorname{mean}\left[(y-\hat{y})^{2}\right]}$ calculated on the validation data. For $4^{\text {th }}$ order polynomial PLS with 20 latent variables, the RMSE is 0.18. Both the polynomial order and the number of latent variables have been chosen to minimize the RMSE.

The 2-dimensional ODP projection of validation data together with the estimated concentration trajectory modeled by $5^{\text {th }}$ order polynomials, are shown in Figure 3. RMSE for CRA is 0.096 (10 parameters in the polynomial model). For inverse regression with $5^{\text {th }}$ order polynomial fit, the RMSE is slightly worse, 0.11 (20 parameters).

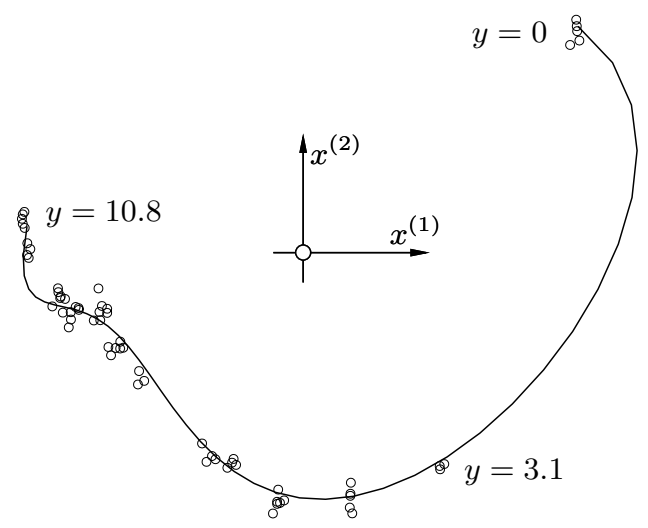

Figure 3: 2-dimensional ODP subspace with validation measurements and $5^{\text {th }}$ order polynomial fit.

\subsection{Leave-Class-Out Validation}

Now exactly all measurements on $3.7 \mathrm{mM}$ are used for validation. For $\left(1^{\text {st }}\right.$ order polynomial) PLS with 10 latent variables (best choice), the RMSE is 0.25. Regression in a 2-dimensional ODP subspace with the inverse regression model and $5^{\text {th }}$ order polynomial, gives RMSE 0.11 (20 parameters). For CRA with $8^{\text {th }}$ order polynomials the RMSE is 0.043 (16 parameters).

\subsection{Discussion}

Using the ODP subspace, the regression can be conducted in 2 dimensions and still give better result than PLS that needs 10-20 dimensions to give reasonable accuracy. Furthermore, the classical regression gives slightly better results compared to the inverse. 


\section{Conclusions}

It is well known that the utilization of structures in data can improve regression. In this work, natural data clustering has been used by the pattern recognition algorithm Optimal Discriminative Projection (ODP) to generate linear subspaces where regression is facilitated. For some nonlinear problems this approach outperforms polynomial PLS.

However, in practice the subspace generated by the ODP often contains a very nonlinear measurement trajectory. It has been found that this nonlinear trajectory is better modeled by a polynomial $\phi$ in the classical regression model $x_{i}=\phi\left(y_{i}, \eta\right)+\epsilon_{i}$, instead of a polynomial $f$ in the inverse regression model $y_{i}=f\left(x_{i}, \theta\right)+e_{i}$. The drawback of the classical regression approach is that it requires more calculations.

On real sensor data from the electronic tongue with a leave-class-out validation procedure, the ODP together with a classical polynomial regression model gave the regression error 0.043 , which should be compared to the best by polynomial PLS: 0.25 - an improvement by factor $>5$.

\section{References}

[1] B. Ari and H. A. Güvenir. Clustered linear regression. Knowledge-Based Systems, (15):169-175, 2002.

[2] A. J. Burnham, J. F. MacGregor, and R. Viveros. A statistical framework for multivariate latent variable regression methods based on maximum likelihood. Journal of Chemometrics, 13:49-65, 1999.

[3] C. Eisenhart. The interpretation of certain regression methods and their use in biological and industrial research. Ann. Math. Statist., 10:162-186, 1939.

[4] G. Camps-Valls et al. Cyclosporine concentration prediction using clustering and support vector regression methods. Electronic Letters, 38(12):568-570, June 2002 .

[5] J. H. Friedman and J. W. Tukey. Projection pursuit regression. Journal of the American Statistical Association, 76:817-823, 1981.

[6] K. Fukunaga. Introduction to Statistical Pattern Recognition. Academic Press, 2nd edition, 1990.

[7] A. R. Gallant. Nonlinear Statistical Models. John Wiley \& Sons, 1987.

[8] G. H. Golub. Matrix Computations, 3 ed. Baltimore : Johns Hopkins Univ. Press, 1996.

[9] I. S. Helland. On the structure of partial least squares regression. Commun. Statist. Simulation, 17:581-607, 1988.
[10] B. Hoadley. A Baysian look at inverse linear regression. Journal of the American Statistical Association, 65:356-369, 1970.

[11] S. Holmin, P. Spångeus, C. Krantz-Rülker, and F. Winquist. Compression of electronic tongue data based on voltammetry - a comparative study. Sensors and Actuators, B(76):455-464, 2001.

[12] W. G. Hunter and W. F. Lamboy. A Baysian analysis of the linear calibration problem. Technometrics, 23:323-350, 1981.

[13] R. A. Johnson and D. W. Wichern. Applied Multivariate Statistical Analysis. Prentice Hall, Upper Saddle River, New Jersey, 4th edition, 1998.

[14] R. C. Krutchkoff. Classical and inverse regression methods of calibration. Technometrics, 9:425-439, 1967.

[15] T. Kubokawa and C. P. Robert. New perspectives on linear calibration. Journal of Multivariate Analysis, 51:178-200, 1994.

[16] Ker-Chau Li. Sliced inverse regression for dimension reduction. Journal of the American Statistical Association, 86(414):316-327, 1991.

[17] H. Martens and T. Näs. Multivariate Calibration. John Wiley \& Sons, Chichester, 1989.

[18] P. Spångeus. New Algorithms for General Sensors. PhD thesis, Linkpings universitet, 2001.

[19] J. L. Plessis and A. J. van der Merwe. A Baysian approach to multivariate and conditional calibration. Computational Statistics \& Data Analysis, 19:539-552, 1995.

[20] D. Di Ruscio. A weighted view on the partial least-squares algorithm. Automatica, 36:831-850, 2000.

[21] Y. Sam and J. Wang. Statistical modeling via dimension reduction methods. Nonlinear Analysis, Theory, Methods \& Applications, 30(6):3561-3568, 1997.

[22] P. Stoica and M. Viberg. Maximum likelihood parameter and rank estimation in reduced-rank multivariate linear regressions. IEEE Transactions on Signal Processing, 44(12):3069-3078, 1996.

[23] H. Takeuchi. A generalized inverse regression estimator in multi-univariate linear calibration. Commun. Statist.-Theory Meth., 26(11):2645-2669, 1997.

[24] S. Wold, N. Kettaneh-Wold, and B. Skagerberg. Nonlinear PLS-modeling. Chemometrics and Intelligent Laboratory Systems, 7:53-65, 1989.

[25] S. Wold, H. Martens, and H. Wold. The Multivariate Calibration Problem in Chemistry Solved by the PLS Method. Springer Verlag, Heidelberg, 1983.

[26] S. Wold, A. Ruhe, H. Wold, and W. J. Dunn. The collinearity problem in linear regression. The partial least squares approach to generalized invereses. SIAM J. Sci. Stat. Comput., 5:735-743, 1984. 